
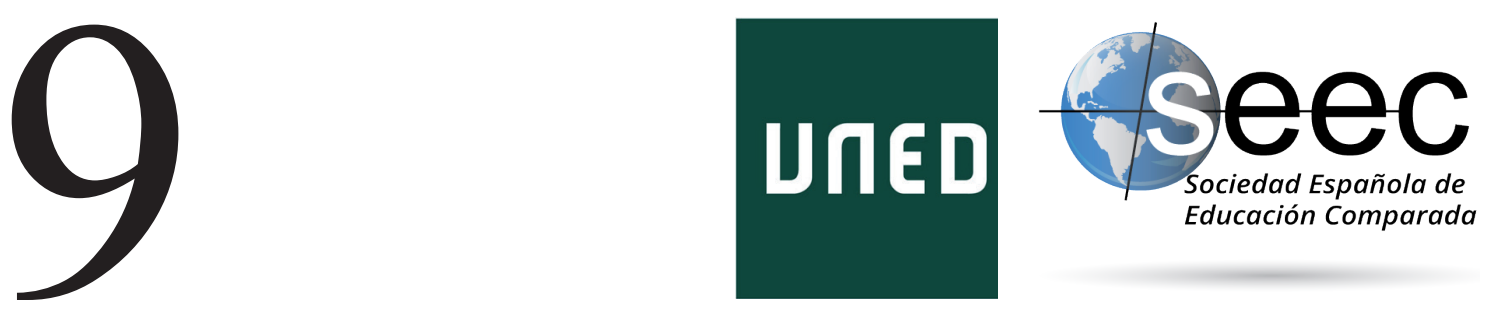

\title{
Capitalismo académico digital
}

Digital academic capitalism

\section{Geo Saura*; Katia Caballero**}

DOI: $10.5944 /$ reec.37.2021.27797

\section{Recibido: 26 de junio de 2020 Aceptado: 16 de octubre de 2020}

\footnotetext{
*Geo Saura: Investigador y Profesor de Política Educativa en la Universidad de Granada. Su investigación está centrada en la neoliberalización de la educación. Investigador Principal del Proyecto «Redes políticas de gobernanza filantrópica: corporaciones, gobiernos y donantes ensamblando, movilizando y mutando las políticas en educación» (REPOGOFI) (Referencia: PPJIA2019-07). Datos de contacto: e-mail: geosaura@ugr.e

**Katia Caballero: Profesora Titular en el Departamento de Didáctica y Organización Escolar de la Universidad de Granada. Su investigación gira en torno a la Educación Superior, la identidad, el desarrollo profesional y el liderazgo pedagógico. Es Investigadora Principal del Proyecto «La influencia del neoliberalismo en las identidades académicas y en la satisfacción profesional» (NEOACADEMIC) (Referencia: PID2019-105631GAIoo). Datos de contacto: e-mail: kaballero@ugr.es
} 


\title{
Resumen
}

El presente artículo examina los procesos que describen la digitalización de la Educación Superior. El principal objetivo es analizar cómo el capitalismo digital, el gobierno de la digitalización, el poder de las métricas digitales y las plataformas académicas están cambiando la producción del conocimiento académico y la vida profesional de los académicos. Para explicar el capitalismo académico digital como marco actual que hace competir a las universidades en el mercado global, realizaremos una revisión de los conceptos que han modificado completamente las normas y cultura universitarias, tales como economía del conocimiento, capitalismo académico, gobierno de los números y racionalidad neoliberal de la performatividad. Seguidamente, estudiaremos cómo los rankings entre universidades y los sistemas de rendición de cuentas se han convertido en formas de control cuantificado que han socavado el propósito original de las universidades. A continuación, analizaremos una nueva forma de capitalismo y gobierno académico centrado en la digitalización como modelo más avanzado de cambio en la Educación Superior. Se comentarán los índices de impacto más importantes y las métricas digitales alternativas (almetrics) para entender la cultura métrica que comienza a instaurarse en las universidades. Finalmente, se estudiará el rol de las plataformas académicas sociales como "Academia.edu" y "ResearchGate" en el contexto del capitalismo académico digital y su influencia en los sujetos académicos, ahora cuantificados y digitalizados.

Palabras clave: Educación Superior; capitalismo digital; métricas; académicos/as; Academia.edu; ResearchGate.

\begin{abstract}
This article examines the processes involved in the digitalisation of Higher Education. The main aim is to analyse how digital capitalism, governing by digitisation, the power of digital metrics and the academic networks are making changes in the production of academic knowledge and in the academics' professional lives. To explain the digital academic capitalism as the current framework that make compite universities in the global market, we will make a review on concepts that have completely modified the academia rules and culture, such as economy of knowledge, academic capitalism, governing by numbers and the neoliberal rationality of performativity. Next, we will focus on how rankings among universities and accountability systems have become ways of quantified control that have undermined the original purpose of universities. Subsequently, we will analyse the new form of academic capitalism and governing centred on digitisation as a step forward in the change of Higher Education. The most important impact indexes and digital alternative metrics (almetrics) will be revised to understand the new metric culture in the academia. Finally, we will study the role of social academic platforms as "Academia.edu" and "ResearchGate" in the context of digital academic capitalism and its influence in the academic subjects that become quantified and digitalised.
\end{abstract}

Keywords: Higher Education; digital capitalism; metrics; academics; Academia.edu; ResearchGate. 


\section{Introducción}

La sociedad digital en la que vivimos está produciendo transformaciones en todas las esferas sociales e institucionales (Lupton, 2015). Estos cambios están teniendo su particular efecto en las universidades y en la relación entre la producción del conocimiento y la cultura académica. Por un lado, los procesos de digitalización modifican el funcionamiento de las universidades que se describían desde las narrativas de la economía del conocimiento (Olssen y Peters, 2005) y de las prácticas políticas del capitalismo académico (Jessop, 2017; Slaughter y Leslie, 2001). Todo ello se debe a que ahora tanto las lógicas de las economías del conocimiento como las del capitalismo académico están siendo sustituidas por las dinámicas propias del capitalismo digital (Fuchs, 2019; Peters, 2020). Por otro lado, los procesos de digitalización social modifican las lógicas del gobierno de los números (Rose, 1991; 1999) que han dominado la academia y han venido transformando la identidad profesional de los académicos durante las últimas décadas. Ahora, al gobierno de los números y a la cultura de la performatividad (Ball, 2012) se le suma el «poder de las métricas» (Beer, 2016), que viene acompañado de procesos de auto-monitoreo y auto-promoción de los «sujetos cuantificados» (Lupton, 2016). Esto da lugar a los nuevos «académicos digitalizados» (Lupton, Mewburn y Thompson, 2018).

El objetivo de este artículo es explorar algunos de los aspectos más relevantes de la digitalización en la Educación Superior para demostrar cómo la producción del conocimiento académico está siendo dominada por el capitalismo digital, el gobierno de la digitalización, el poder de las métricas y las plataformas sociales académicas. Es lo que llamamos «capitalismo académico digital». Para ello, tras esta sección introductoria, el artículo se divide en seis apartados.

En primer lugar, se describe cómo desde la década de los 90 las universidades han sido dominadas por la economía del conocimiento, el capitalismo académico, el gobierno de los números y la racionalidad neoliberal de la cultura de la performatividad. Seguidamente, se analiza cómo los rankings de universidades y las políticas de rendición de cuentas se fueron hegemonizando como los cambios políticos más determinantes para modificar el funcionamiento de las universidades bajo los principios del capitalismo académico desde comienzos del siglo XX. Los rankings y los sistemas de rendición de cuentas han ejercido un control directo sobre las universidades y los académicos, alterando las formas de producción del conocimiento científico. En tercer lugar se aborda la noción de capitalismo digital y el gobierno de los dígitos liderados por los procesos algorítmicos de los Big Data (Kitchin, 2014), el capitalismo de vigilancia (Zuboff, 2019) y la «ubersificación de la universidad» (Hall, 2016). A continuación se describen los índices de impacto de Journal Citation Reports (JCR), Scimago Journal Rank (SJR), h-Index de Google Scholar y las métricas alternativas digitales (almetrics) como principales sistemas para metrificar digitalmente la producción del conocimiento y generar la cultura métrica académica (Burrows, 2012). Finalmente, se analizan las plataformas sociales académicas más importantes -Academia.edu y ResearchGate- y su legitimización dentro del capitalismo académico digital, fomentando la construcción de sujetos académicos cuantificados y digitalizados (Lupton, Mewburn y Thompson, 2018). Se concluye haciendo referencia a las nuevas funciones asumidas por los académicos de auto-monitoreo y auto-promoción para mejorar el impacto métrico como cuidado del sí profesional y su influencia en la generación de nuevas identidades académicas digitales. 


\section{El capitalismo académico y el gobierno de los números}

El conocimiento generado desde las universidades se ha convertido en una forma de producción para avanzar en las lógicas competitivas de las economías globales (Olssen y Peters, 2005). Basándose en los principios de la economía del conocimiento, término que comienza a expandirse a finales del siglo XX, el discurso internacional ha conducido a las instituciones universitarias a adaptarse a los cambios del capitalismo global, haciendo del conocimiento una forma de competición económica entre países. Los informes de la Organización para la Cooperación y el desarrollo Económico (OCDE, 1996a; 1996b; 1996c) fueron unos de los primeros discursos supranacionales que impulsaron a las universidades a convertir el avance del conocimiento en una forma de competir en el mercado global. En el contexto europeo, la adhesión de las universidades a la economía del conocimiento se materializa con la creación del Espacio Europeo de Educación Superior (EEES). El EEES fue la estrategia más pragmática para homogeneizar progresivamente a las universidades europeas con la finalidad de que estas pudieran competir con las economías de China y Estados Unidos.

De esa relación entre producción de conocimiento y competición, propia de una lógica capitalista, nace el concepto de «capitalismo académico» (Slaughter y Leslie, 2001). La labor académica se transforma así, en las últimas tres décadas, en una actividad al servicio del capitalismo regida por principios de producción y competitividad económicos (Brunner et al., 2019; Slaughter y Rhodes, 2004). El resultado ha sido la conversión de la academia en un espacio donde se desarrollan prácticas y dinámicas de producción del conocimiento, entendido como mercancía. En este contexto, el capitalismo académico se sirve del capital humano de las universidades para potenciar al máximo la producción. De ahí que los cambios que ha sufrido la profesión académica, orientada a aumentar la producción de resultados de investigación y la posición en los rankings entre universidades, haya fomentado la competición por recursos externos de financiación y la sobreproducción del conocimiento.

El capitalismo académico ha dado lugar a nuevos sujetos académicos gobernados por los números (Rose, 1991). El gobierno a través de números y estadísticas cuantificadas ha expandido «tecnologías políticas» que se sustentan en la producción, la autorización, la validación, la difusión y la acumulación (Rose, 1999). Estos procesos de gobernanza mediante números han sido impulsados desde la política educativa global para clasificar, comparar y categorizar a las instituciones universitarias (Grek, 2009). Todo ello ha generado un sentimiento de dependencia de los números (Sellar, 2015) que queda justificado por las propias políticas educativas como la forma de controlar la calidad, generar verdad, legitimar relaciones de poder y constituir los nuevos sujetos académicos neoliberales contemporáneos (Ball, 2012). Esta gobernanza académica neoliberal basada en la cuantificación (Lewis, 2013) ha llevado a las universidades a una sobreproducción de conocimiento regida por una de las máximas del capitalismo académico: «publicar o perecer» (Harzing, 2010). La incesante producción de artículos como forma de supervivencia en la universidad ha generado las nuevas subjetividades académicas propias del «homo economicus» (Read, 2009). Así, las subjetividades se construyen cuando el académico se sumerge en las dinámicas de producción, ejerce un auto-control sobre ellas y asume la responsabilidad de sus éxitos y fracasos. Esta forma de subjetivación es 
propia de la gubernamentalidad neoliberal en la Educación Superior y transforma a los académicos en empresarios de sí mismos.

Estas lógicas de la economía del conocimiento que funcionan por medio del capitalismo académico y la gobernanza de los números han producido variaciones epistemológicas sobre cómo y para qué se produce el conocimiento en las universidades. La performatividad, que fue un concepto que abordó el filósofo francés Jean-François Lyotard (1984) en «La condición postmoderna. Informe sobre el saber», ha sido un posicionamiento teórico que ha servido para comprender los principios y los cambios que acompañan a la producción del conocimiento mediante dinámicas de rendimiento contante en la producción de resultados. Los primeros trabajos que comenzaron a utilizar la noción de performatividad en la educación superior, que han venido desde los estudios en educación comparada (Barnett, 1996; Cowen, 1996), se centraron en demostrar cómo, ya en la década de los 90, las universidades tenían como meta luchar por la economía del conocimiento a través de prácticas que hacían que su capital humano orientase la actividad académica hacia dinámicas de constante rendimiento cuantificado. La cultura de la performatividad persigue la optimización relacional de reciprocidad input/output sustentada en la maximización de resultados. De ahí que performatividad se base en el determinismo y en la filosofía positivista de la eficiencia. Las acciones que generan la cultura de la performatividad en la universidad están enfocadas a la producción de conocimiento como valor económico por encima de cualquier otro valor. Esta cultura modifica la relación del académico con su actividad profesional, con sus colegas y, lo más importante, consigo mismo. La performatividad, que se basa en la cuantificación de la producción, ha sido muy atractiva y eficaz para consolidarse como tecnología de gobierno que convierte a los números en garantes de la verdad y de la calidad. De ahí que los sistemas de comparación, a través de rankings, se hayan servido de estos procesos de gobernanza numérica para determinar cuán válido es un académico, una universidad y un país.

\section{Rankings y rendición de cuentas}

El gobierno de los números ha originado políticas para reorientar las formas de producción del conocimiento académico y las lógicas de funcionamiento de las universidades. Dichas políticas se han materializado a través de la consolidación de rankings universitarios (Shore y Wright, 2015) y evaluaciones basadas en la rendición de cuentas (accountability) (Cannizzo, 2015).

Aunque los rankings de universidades se remontan a la década de los años 80, la expansión de los mismos se produce a principios de la primera década del siglo XX (De Angelis y Harvie, 2009; Shore y Wright, 2015). El Academic Ranking of World Universities (ARWU, por sus siglas en inglés), conocido también como Ranking de Shanghái, el ranking Times Higher Education World University (THE) y el QS World Rankings (que incorpora a diferentes rankings pertenecientes a la corporación británica Quacquarelli Symonds) se han consolidado durante la última década como los tres sistemas de clasificación más importantes de la política educativa global. Estos rankings competitivos han normalizado que la finalidad de las universidades sea mejorar constantemente los resultados numéricos de los indicadores de producción del conocimiento para situarse en el mejor posicionamiento de las clasificaciones. El ARWU o ranking de Shanghái, que comienza en el año 2003, es el más relevante de todas las clasificaciones en las que se posicionan a unas universidades y regiones respecto a otras. Aunque hay múltiples 
dimensiones de medición de la calidad de las universidades, el $60 \%$ del valor total de los indicadores de ARWU se fundamentan en torno a publicaciones de impacto en revistas indexadas. El ranking de Shanghái ha legitimado la cultura de rankings mediante la cual los países y las universidades son auto-gobernadas en procesos de competencia. Esta cultura hegemónica de los rankings para medir la calidad en función de la producción, ha tenido grandes detractores en la literatura internacional (Lynch, 2013; Shore y Wright, 2015). Una de las corrientes críticas más relevante es la que se está elaborando con base en el pensamiento descolonial (Leite, 2010; Shahjahan y Blanco-Ramírez, 2016). El pensamiento descolonial es una corriente epistémica y política que se basa en la crítica de la cosmovisión de la modernidad que hegemoniza el conocimiento occidentalizado en la educación (Grosfoguel 2008). Esta corriente analiza cómo las universidades del sur global han ido modificando sus culturas de origen a través de un pensamiento colonizado impuesto como único enfoque para comprender la realidad (Lander, 2000; CastroGómez y Grosfoguel, 2007). Son procesos de hegemonización que expanden modos de entender, de ser y de representar los fenómenos (Mignolo, 2011) a través de un «epistemicidio» en la producción del conocimiento (Santos, 2010). Estos cambios en la producción del conocimiento tienen que ver con la imposición de las dinámicas del capitalismo académico que han alejado a las instituciones universitarias de sus culturas originarias. Las universidades del sur global han vivido procesos de ruptura abrupta con sus contextos de origen para dirigir la producción del conocimiento en una adaptación a las lógicas competitivas del mercado global por las que han sido colonizadas. Estos cambios epistémicos en las instituciones de educación superior de América Latina han llevado a que se generen procesos de resistencia a la hegemonía de la economía del conocimiento y del capitalismo académico por medio de una epistemología del sur que tiene como fin último decolonizar el saber (Castro-Gómez y Grosfoguel, 2007; Santos, 2010).

La cultura de la auditoría (Power, 2000), bajo la que se sostienen los rankings universitarios, se instaura gracias a las políticas de rendición de cuentas lideradas por un gobierno de los números que hace recaer en los sujetos académicos toda la responsabilidad sobre la producción. Para ello, durante las últimas décadas, a través de los sistemas de evaluación, se han impulsado estrategias para lograr que el capital humano de las universidades produzca conocimiento constantemente medido en rendimientos de productividad de los resultados de investigación. Estos resultados se materializan principalmente en publicaciones, captación de recursos de investigación financiados de modo competitivo y transferencia de resultados al mundo empresarial. Las políticas de rendición de cuentas basadas en la producción se han analizado en diferentes países y dan muestras de cómo se legitima la competitividad individual bajo nuevos espacios de control en la academia (Cannizzo, 2015; Fardella, Sisto, Jiménez, 2017; Jankowski y Provezis, 2014). Uno de los discursos supranacionales iniciales para promover políticas basadas en el rendimiento de cuentas viene de la mano del Banco Mundial a finales de los años 90, periodo en el que se instaura la noción de economía del conocimiento para competir por el conocimiento global. En el informe del Banco Mundial "The financing and management of higher education" (Johnstone, Arora y Experton, 1998), la lógica discursiva orienta a los países a instaurar modelos de mercado en las universidades para que los académicos perciban salarios e incentivos económicos en función de su desempeño y producción. Aunque las políticas de rendición de cuentas forman parte de una corriente global, en cada contexto se ha desarrollado de manera diferente, pues se han tenido que ajustar a las tradiciones culturales de las universidades en cada país (modelo de mercado, 
humboldtiano y napoleónico). Tomando como ejemplo el caso español y en línea con la creación del Espacio Europeo de Educación Superior (EEES), en el año 2002 se crea la Agencia Nacional de Evaluación de la Calidad y Acreditación (ANECA) para poner en marcha dinámicas de rendición de cuentas basadas en la producción científica. Se crean así los sistemas de acreditación para acceder a distintas figuras de personal docente y los de incentivación económica a través de los sexenios de investigación. La finalidad de la ANECA, desde su creación, fue valorar la calidad del trabajo académico según variables cuantitativas basadas en la producción de conocimiento bajo dinámicas propias del capitalismo académico (Amigot y Martínez, 2013; Díez-Gutiérrez, 2018; Gómez, Jódar y Bravo, 2015; Manzano, 2015). En el año 2015 y en el año 2019 se establecen dos nuevas políticas que hacen que la ANECA valore esencialmente la producción del conocimiento a través de artículos científicos indexados en las industrias de Web of Science y Scopus ${ }^{1}$. Así, en las universidades españolas, el personal docente ha tenido que adaptarse durante la última década a dinámicas descontroladas de rendición de cuentas que tienen como resultado la sobreproducción de conocimiento y el sometimiento al capitalismo académico. Estos cambios abruptos en la política universitaria española han llevado de modo reciente al desarrollo de estudios y trabajos de investigación que destacan los efectos de la racionalidad neoliberal y el gobierno de los números en las universidades y en la actividad profesional de los académicos (González-Calvo y Arias-Carballal, 2018; Saura y Bolívar, 2019; Vayreda, Conesa, Revelles-Benavente, González-Ramos, 2019).

\section{Hacia un capitalismo académico digital}

Si bien la economía del conocimiento ha servido para comprender las lógicas del capitalismo académico en la década de los 90, la noción de capitalismo académico digital es ahora más apropiada para abordar el nuevo rumbo del sistema capitalista hacia la producción y mercantilización de conocimiento digitalizado. Schiller (2000) comenzó a utilizar el concepto de capitalismo digital para describir las variaciones del mercado global en torno a medios de producción digitales al tiempo que se expandía el uso de Internet (Fuchs, 2019; Schiller, 2000). Es común también utilizar otros términos como «capitalismo informacional», "capitalismo de redes» (Fuchs y Mosco, 2015), «capitalismo cognitivo» o "capitalismo del conocimiento» (Peters, 2020; Peters y Jandrić, 2018) para hacer referencia a las formas de producción del conocimiento en la era de la digitalización. En lugar de recurrir a la noción de economía del conocimiento, como se hacía durante las últimas tres décadas, ahora, discursos supranacionales como los de la Organización para la Cooperación y el Desarrollo Económico (OCDE, 2020) y la Comisión Europea (2020), se decantan por hablar de «economías digitales» o «economía digital competitiva» para supeditar la producción del conocimiento a los principios del capitalismo digital. La idea esencial del capitalismo digital es que la acumulación del capital y las formas de producción se desarrollan sustancialmente a través de un trabajo inmaterial producido por la tecnología digital. Así, los procesos de producción digitalizados se configuran a través del uso de redes y plataformas, tipo Uber, Airbnb, LinkedIn

1 ANECA. Real Decreto 415/2015, de 29 de mayo, por el que se modifica el Real Decreto 1312/2007, de 5 de octubre, por el que se establece la acreditación nacional para el acceso a los cuerpos docentes universitarios. En 2017 y 2020 se establece nuevos criterios para la acreditación basados en la producción cuantitativa de resultados de investigación materializados en la primacía de las revistas de web of science y scopus. 
y otras similares, y aunque algunas investigaciones se refieren a ellas como parte de una «economía colaborativa» (Sundarajan, 2016), para otros autores más críticos, como Srnicek (2017), forman parte del «capitalismo de plataformas».

Como consecuencia de lo anterior, comienzan a proliferar los análisis que se sirven del abordaje teórico del capitalismo digital para examinar los cambios más relevantes en la Educación Superior. Hall (2016) se ha centrado en las dinámicas internas de las universidades a través de la producción del capital y los espacios de la digitalización académica a través de la noción de la «Ubersificación». La ubersificación de la universidad es una metáfora para comprender que el funcionamiento de la universidad actual está basado en lógicas del capitalismo digital que se materializan a través de la mercantilización del conocimiento en plataformas como la del magnate tecnológico Uber. Además, las lógicas del capitalismo digital permiten comprender la influencia que la industria de las corporaciones tecnológicas de Sillicon Valley ejerce sobre la reforma global de la Educación Superior (Means, 2019). Junto a ello, los estudios que abordan la digitalización de la Educación Superior comienzan a apostar por la noción del capitalismo digital a través de la corriente de análisis de la economía política (Mirrlees y Alvi, 2020).

El capitalismo digital genera la transformación de la producción del conocimiento en las universidades, de manera que lo que antes estaba gobernado por números, paulatinamente comienza a estar gobernado por la digitalización. El gobierno de los números que auspiciaba la cultura de la performatividad ha devenido en la datificación, que consiste en procesar los números para convertirlos en datos cuantitativos y analizarlos por medio de tecnologías digitales (Williamson, 2018). Estos procesos de datificación fomentan que vivamos gobernados por la digitalización. La digitalización es el tratamiento de los datos analógicos y su conversión en dígitos a través de códigos binarios que pueden ser procesados por algoritmos y softwares. Estos procesos de digitalización están haciendo que el trabajo académico esté sufriendo nuevas modificaciones, a la vez que las universidades son objeto de las dinámicas expansivas del mercado global del conocimiento digitalizado. El gobierno de los dígitos está generando importantes cambios en la producción del conocimiento en las universidades gracias a las posibilidades que las grandes corporaciones tecnológicas digitales ofrecen por medio de los Big Data y la inteligencia artificial (Kitchin, 2014; Mayer-Schönberger y Cukier, 2013). Big data hace referencia a las cantidades masivas de datos que son procesados en grandes volúmenes y a una velocidad de análisis muy elevada para ofrecer valores relacionados con el comportamiento de los sujetos frente a la información que le ofrecen los medios tecnológicos. Estos cambios que devienen de los procesos de almacenamiento de datos masivos se recogen de modo cotidiano para extraer patrones de conducta exhaustivos, de gran alcance y flexibles que son almacenados y procesados a tiempo real (Kitchin, 2014; Williamson, 2019). Desde la visión de Mayer-Schönberger y Cukier (2014) la aplicación de los Big Data en el campo de la educación, junto a la inteligencia artificial, componen unos de los cambios más importantes en la política educativa global (Pedró, Subosa, Rivas y Valverde, 2019). A través de esta tipología de procesamiento digital, se obtiene información personalizada e individualizada de los procesos de aprendizaje a tiempo real, por medio de sistemas probabilísticos que prevén aciertos y errores a la vez que conducen las acciones hacia unas metas que son en parte predeterminadas. En este sentido, la selección de información por parte del sujeto condiciona sus elecciones futuras, pues la tecnología digital se encarga de establecer filtros en función de los patrones de búsqueda del sujeto, y esto puede hacerlo mediante cualquier dispositivo y desde cualquier lugar. 
Con referencia concreta a los procesos de control digitalizados, Shoshana Zuboff (2019) introduce el término "capitalismo de vigilancia», y las prácticas que se derivan del mismo forman lo que Lyon (2018) denomina «cultura de vigilancia». El «capitalismo de vigilancia» se entiende como el conjunto de prácticas de control del sistema capitalista sobre la información publicada en redes digitales y sobre las dinámicas de acceso de los sujetos a ella. Por su parte, la cultura de vigilancia hace referencia a las prácticas y compromisos que los sujetos asumen en el control de sus dígitos. De esta manera, los académicos se convierten en «trabajadores del conocimiento digitalizado» (Lupton, 2015, p. 66), es decir, orientan sus esfuerzos a la máxima difusión de su producción para que esta revierta en ellos en forma de dígitos y, por ende, de prestigio digital.

Para analizar la transformación de las universidades a través del capitalismo digital y del gobierno de la digitalización, destacaremos el papel de las métricas, así como el de las plataformas y redes sociales académicas. Se revisarán los índices de factor de impacto de la producción científica en Web of Science y Scopus, el «h-Index» de Google Scholar, el código Orcid, la industria de las métricas de impacto digitales de las publicaciones científicas de las revistas y las métricas alternativas (Altmetrics), así como las plataformas y redes sociales académicas de publicaciones como Academia.edu, ResearchGate, Twitter y LinkedIn.

\section{Métricas digitales}

Los procesos de digitalización social han hecho que las métricas digitales sean parte de nuestras vidas (Beer, 2015; Lupton, 2015). A través de cualquier dispositivo tecnológico digital se ha normalizado que metrifiquemos las horas de sueño, las calorías que consumimos o los pasos que andamos. Lo que medimos y la importancia que otorgamos a los resultados que obtenemos afecta a lo que hacemos y a lo que somos. Las métricas influyen en la elaboración y modificación de juicios sobre nosotros mismos y en las consecuencias de esos juicios en nuestras vidas (Beer, 2015). Las métricas digitales son procesos de medición a través de datos masivos que han sido, desde la última década, el mecanismo más empleado para la generación de valor del capitalismo digital contemporáneo (Shiller, 2015). Es decir, la digitalización métrica es el medio de la datificación masiva para generar los valores del capitalismo digital. Estas métricas digitales, que se configuran a través de los Big Data, están cambiando las formas de gobernar a los sujetos y los espacios, de administrar a las organizaciones, de hacer funcionar las economías y de desarrollar ciencia (Kitchin, 2015). Así, las métricas se han convertido en nuevos espacios de medición digitalizada a través de códigos, softwares y algoritmos que están configurando nuevos procesos de control y de gobierno de los sujetos.

Debido a estos procesos de digitalización social, la universidad comienza a quedar dominada por nuevos mecanismos de gobernanza basados en el «poder de las métricas» o «cultura métrica» (Beer, 2016; Burrows, 2012; Feldman y Sandoval, 2018; Williamson, Bayne y Shay, 2020). Los factores de impacto de Web of Science y Scopus, el «h-Index» de Google Scholar y las múltiples métricas digitales de las revistas de impacto (denominadas altmetrics o métricas alternativas) se han normalizado como procesos de gobierno a través de mediciones digitalizadas en códigos y softwares. Estas métricas digitales que están dominando la producción del conocimiento académico se constituyen como los procesos más avanzados de la industria de la bibliometría. La bibliometría nace como una ciencia fundamentada en la aplicación de las matemáticas y métodos estadísticos a 
las publicaciones científicas como un neologismo que hibrida los términos análogos de «biometría, econometría y cienciometría» (Pritchard, 1969, p. 348).

El índice de impacto Science Citation Index (ISI) de Web of Science, perteneciente ahora a Clarivate Analitics, que fue en su inicio la propiedad intelectual y científica de Thomson Reuters, nace en 1961 y se materializa mediante los impactos de revistas en Journal Citation Reports (JCR). El índice de impacto SCimago Journal Rank (SJR) de Scopus, propiedad de Elsevier mide los factores de impacto de las publicaciones de las revistas desde 1998 a través del algoritmo de Google PageRank. Tanto JCR como SJR se basan en la medición de las citaciones de las publicaciones científicas en revistas académicas a través de cuartiles según la importancia que dichas revistas tienen por su nivel métrico de citación o impacto. Por otro lado, el h-Index de Google Scholar, fundado por Hirsch (2005), se basa en un impacto de citación de cada científico mediante la relación entre el número de citas y los artículos publicados que posee. Tanto los índices de impacto de revistas JCR y SJR, así como el índice de impacto de cada académico en Google Scholar (h-Index) se han convertido en una finalidad misma de la producción científica. Son espacios de gobierno en los que la academia (investigadores y revistas) aspira a introducirse de modo exitoso a través del aumento de citaciones de impacto. Esto es así porque en la academia jugamos al juego de las métricas, a la vez que las métricas juegan con nosotros. Todo ello ha derivado en procesos de auto-gobierno que nos hacen actuar siguiendo sus reglas y sus límites, y convirtiéndonos en sujetos digitalizados.

Como medio alternativo a las tres grandes bases de datos que miden el impacto de las publicaciones (JCR, SJR y Google Scholar), en la última década aparecen las métricas alternativas o altmetrics. Las almetrics son métricas digitales del impacto de las publicaciones en la Web a través de blogs, menciones en medios de comunicación, Twitter, Facebook, y demás redes sociales, así como de las veces que se referencian las publicaciones en Wikipedia o la cantidad de veces que un artículo es guardado en repositorios como Mendeley. Si bien las altmetrics tienen su origen en la webometría de los años 90 (Torres-Salinas, 2013), se consolidan en la última década como registros de atención para cuantificar cuántas personas han descargado un artículo. Consiste en medir la difusión para comprender dónde y por qué se está discutiendo y compartiendo una investigación; se trata de un indicador de influencia para reconocer efectos tangibles de los avances que producen los resultados de las publicaciones en la sociedad (Almetric, 2020). Las métricas alternativas, aunque nacen como sistemas de medición de impacto alternativos al funcionamiento de JCR, SJR y Google Scholar, en la actualidad se han convertido en una industria más del capitalismo digital para producir y ayudar a la comercialización del conocimiento científico. De esta manera, hoy por hoy se ha normalizado que las webs de las revistas informen sobre todo tipo de métricas digitales para cuantificar la valía de sus publicaciones.

Plum Analytics se funda en 2012 como una de las primeras corporaciones de las altmetrics y, desde el año 2017, forma parte de la industria de la producción del conocimiento en Elsevier (Plum Analytics, 2020). PlumX Metrics es la plataforma de las métricas alternativas de Plum Analytics para medir el impacto de los artículos de las revistas de Scopus. Las métricas digitales alternativas de PlumX Metrics se hacen a través de 5 categorías: Citations o cantidad de veces que se cita un artículo en CrossRef, PubMed, Scielo, Scopus, etc.; Usage a través e la cantidad de descargas, vistos, catálogos, abstracts, reproducciones de vídeo; Captures o capturas de pantallas en marcadores, exportaciones, seguidores, recomendaciones de lectura; Mentions o menciones de un 
artículo en comentarios de revistas, blogs, medios de comunicación; y Social media para medir la cantidad de likes, comentarios y veces que se comparte un artículo en las principales redes sociales como Facebook o Twitter.

Digital Science, una corporación creada en 2010, se ha consagrado como uno de los grandes magnates tecnológicos del capitalismo digital en la medición de la producción del conocimiento científico, mediante las métricas digitales alternativas de impacto. En su web se especifica que es una empresa «que se enfoca en inversiones estratégicas en compañías nuevas que apoyan el ciclo de vida de la investigación» (Digital Science, 2020). Almetric (que funciona de modo similar a PlumX Metrics y por esa razón no será tenido en cuenta aquí) y Dimensions son partes de Digital Science materializadas como plataformas para el análisis de las métricas digitales del impacto científico. Dimensions mide cada artículo en relación a las siguientes categorías: Publications, que es el número de publicaciones relacionadas con la búsqueda; Citations es la cantidad de veces que una publicación ha sido citada por otras publicaciones en las bases de datos; Publications with citations es el número de publicaciones con al menos $n$ citas o publicaciones que aún no se han citado; Field Citation Ratio es un indicador del rendimiento relativo de citas de una publicación en comparación con artículos de edad similar en su área temática para establecer el promedio de impacto; Relative Citation Ratio que indica el rendimiento relativo de citas de una publicación al comparar su tasa de citas con la de otras publicaciones en su área de investigación; y Recent citations que es el valor de las citas recientes a través del número de citas recibidas en los últimos dos años.

Tanto PlumX Metrics, Dimensions y Altmetric se han consolido en las revistas científicas como métricas alternativas que conviven directamente con los factores de impacto de JCR, SJR y Google Scholar. Ahora las revistas utilizan tanto los resultados algorítmicos digitales de PlumX Metrics, Dimensions y Almetric como métricas a través de visualizaciones muy atractivas. Véase a continuación dos ejemplos gráficos de estas altmetrics digitales de los artículos científicos:

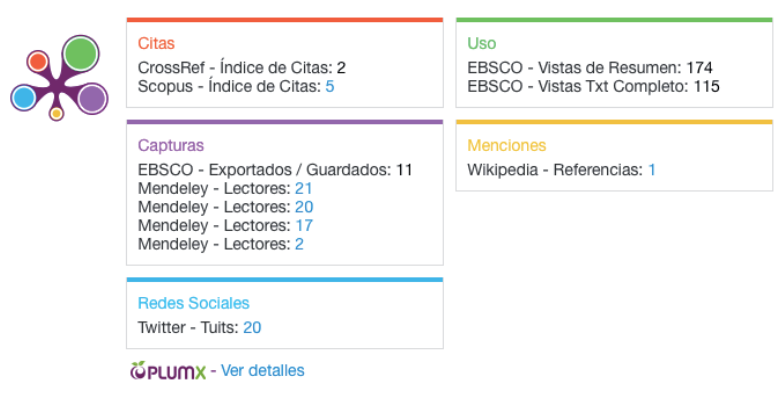

Imagen 1. PlumX Metrics

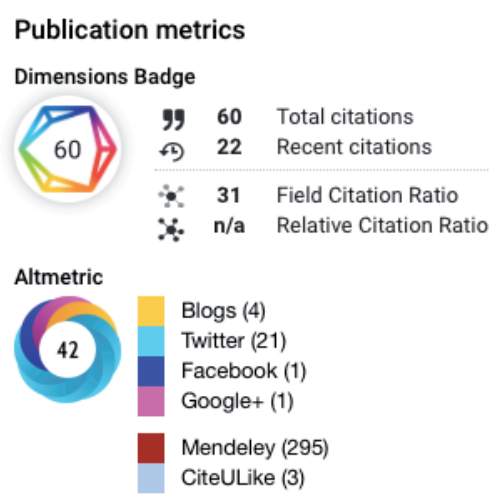

Imagen 2. Dimensions y Almetric

El uso de estas métricas digitales está generando nuevas formas de gobierno de los sujetos académicos y de la producción del conocimiento en las universidades. A través de lentes de análisis foucaultianas, Sharpe y Turner (2018) han definido el uso de estas métricas digitales para la producción de conocimiento académico como una nueva forma de la razón gubernamental neoliberal. Las métricas como forma de gobierno, que tienen su base en la estadística, es algo que Michel Foucault estudió en profundidad desde 1977 
a 1979. La estadística y las métricas funciona como control poblacional por medio de la biopolítica (Foucault, 2007) y como gobierno de sí mediante la gubernamentalidad de las tecnologías del yo (Foucault, 2008). Siguiendo esta base teórica, las métricas digitales hibridan las formas de gobierno del conocimiento científico a través de estructuras de poder externas, así como desde modos de auto-gobierno. Las métricas se han convertido en un fin mismo de las publicaciones por lo que es necesario que cada sujeto se gobierne a sí mismo para, constantemente, alcanzar un mayor impacto. En definitiva, estas métricas han sido legitimadas como espacios de gobierno de la universidad neoliberal a la vez que han constituido la «metrificación de la academia» (Burrows, 2012, p. 356).

\section{Plataformas académicas}

Academia.edu y ResearchGate se han consolidado como las plataformas sociales del ámbito académico. Ambas se fundan en el año 2008 con la finalidad de que cada usuario pueda libremente subir y compartir los resultados de producción científica a la vez que establecen interacciones virtuales en la red académica global. En junio de 2020, Alexa Internet Inc., la compañía de Amazon sobre métricas digitales y análisis de datos masivos del tráfico web, sitúa a ResearchGate en la posición \#168 del Alexa Ranking, mientras que Academia.edu se posiciona en la \#286 entre todas las webs del mundo. La misión de Academia.edu «es acelerar la investigación mundial» de 139 millones de académicos que la conforman, incorporando más de 39 millones de artículos (Ademia.edu, 2020). También mantienen que se utiliza la red «para compartir su investigación, monitorear análisis profundos sobre el impacto de su investigación y rastrear la investigación de los académicos que siguen». Academia.edu ofrece un paquete de visualizaciones de impacto métrico a través de algoritmos que exponen desde qué servidor se está leyendo la investigación, cuántos usuarios están prestando atención y descargando un artículo, así como quiénes están recomendando una publicación determinada. ResearchGate, por su parte, sostiene que la finalidad de la corporación es ayudar a los 17 millones de usuarios académicos que componen la red para "que su progreso sea más rápido» (ResearchGate, 2020). Aunque funciona de modo similar a Academia.edu, el aspecto más distintivo de ResearchGate es el índice de impacto que aporta compuesto por un algoritmo interactivo entre las publicaciones subidas, las citas recibidas de las publicaciones, las interacciones de actividad que cada autor realiza en la web y la cantidad de followers que tiene. El resultado de todo ello es un impacto métrico denominado ResearchGate Score (RG Score). La finalidad del $R G$ Score es analizar toda la investigación de cada usuario y convertirla en una «fuente de reputación» (ResearchGate, 2020). Así, establece un h-Index convertido en una métrica digital más del impacto de la producción científica y citas recibidas mediante un dígito que sitúa a cada usuario en un percentil según los resultados del puntaje del $R G$ Score entre todos los miembros de la red. Análisis desde el campo de la bibliometría y la comunicación han demostrado lo fácil y común que es manipular los resultados e interacciones de estas plataformas para aumentar el $R G S$ core (Orduña-Malea, Martín-Martín y Delgado-López-Cózar, 2016).

En la actualidad, Academia.edu ha recibido 33.8 millones de dólares desde diferentes donaciones de usuarios que pagan por una membresía Premium en la que pueden ver sus datos actualizados constantemente, así como desde fundaciones filantrocapitalistas y corporaciones que donan e invierten con dinámicas de capital de riesgo como Tencent, Khosla ventures, True ventures, Spark ventures y Spark capital. ResearchGate posee un 
fondo total de inversión de 87.6 millones de dólares. Las inversiones de ResearchGate han venido de la mano corporaciones como Groupe Arnault, Benchmark o Goldman Sachs. El filantrocapitalista Bill Gates, que invirtió 35 millones de dólares en la red social académica, se ha convertido en uno de los principales donantes y defensor de ResearchGate. Las inversiones y donaciones que reciben ResearchGate y Academia.edu demuestran la importancia del «filantrocapitalismo», esa hibridación de las lógicas de la filantropía con las del capitalismo (Saura, 2020), en los cambios que está viviendo la producción del conocimiento científico en el capitalismo digital. Gary Hall ha sido tajante para analizar la situación de estas redes sociales académicas como emblemas del capitalismo digital. Centrándose concretamente en «Academia.edu», sostiene que «tiene una relación parasitaria con el sistema de educación pública, en el sentido de que estos académicos ahora trabajan de forma gratuita para ayudar a construir su plataforma privada con fines de lucro al proporcionar el aporte agregado, los datos y el valor de la atención» (Hall, 2015).

Algunas investigaciones recientes analizan cómo estas plataformas sociales académicas son mecanismos del capitalismo digital para construir nuevos sujetos académicos digitalizados y cuantificados (Hall, 2016; Lupton, Mewburn y Thompson, 2018; Tanczer, Deibert, Bigo, Franklin, Melgaco, Lyon, Kazansky y Milan, 2020). Discutiendo la interrelación de la visibilidad en los espacios digitales, las métricas y los nuevos espacios de control, Academia.edu y ResearchGate han sido analizados como los ejemplos más claros de la nueva cultura de la «vigilancia académica» (Tanczer et. al., 2020, p.X). Para ello, y partiendo de las nociones del capitalismo de vigilancia (Zuboff, 2019) y la cultura de la vigilancia (Lyon, 2017), como formas de control avanzadas del capitalismo digital, estas plataformas se han convertido en emblemas de la academia neoliberal a través de la cuantificación y puntuación desenfrenadas (Tanczer et.al, 2020). La inteligencia artificial y los Big Data son los procesos de digitalización que conducen estas plataformas de metrificación digital. Ambas redes sociales académicas utilizan los procesos algorítmicos de datos masivos de Google Analytics. Aunque no hay mucha más evidencia al respecto, sí se conoce que utilizan procesos algorítmicos adictivos similares a los de Facebook (Tanczer et.al, 2020, p. 23).

\section{Conclusiones}

Los procesos de digitalización del conocimiento como mecanismos de producción del capitalismo académico digital que han sido abordados en este trabajo, convierten a los sujetos académicos en empresarios de sí mismos. El académico ya no solo debe investigar y acumular publicaciones, sino que también debe ejercer un cuidado de las mismas, así como de sus seguidores. Las recomendaciones y las interacciones son un valor añadido al éxito de la propia empresa. En este sentido, los procesos de «Uberficación de la universidad» (Hall, 2016) están produciendo sujetos académicos más individualizados, cuantificados y digitalizados (Adell, Castañeda y Esteve, 2018; Hall, 2016). La autopromoción y el cuidado del perfil académico son indispensables para generar una marca personal y garantizar el prestigio académico. Las herramientas de análisis foucaultianas de las tecnologías del yo (Foucault, 1990) y la «ética del cuidado de sí» (Foucault, 2008) han sido útiles para comprender cómo la digitalización de la Educación Superior está generando nuevos sujetos académicos. Así, las plataformas se han convertido en un escaparate, cuyo atractivo depende de las labores de cuidado que asuma el sujeto. De esta forma, los sujetos académicos están sometidos a procesos de auto-gobierno y se 
convierten en «almas académicas cuantificadas» (Hammarfelt, de Rijcke y Rushforth, 2016). La finalidad de la auto-promoción como cuidado del sí digital, se hace a través de la supervisión constante de estos espacios virtuales para intentar aumentar el impacto, el éxito académico y el ego-ideal que conforma la identidad académica. Deborah Lupton (2015) lo concibe como sujeto cuantificado que intenta dar sentido a los espacios de digitalización de lo social.

Las tecnologías digitales funcionan para que cada cual, desde la libertad, recopile, registre, supervise, comparta y vigile su producción científica creando una identidad académica digital. El sujeto académico cuantificado y digitalizado asume prácticas de auto-seguimiento y auto-monitoreo reflexivo. Utiliza las métricas de ResearchGate y Academia.edu para ampliar las interacciones virtuales, los impactos y el cuidado de su propia empresa. Para actualizar la expresión de Harzing (2010) de «publicar o perecer» que da sentido a las lógicas del capitalismo académico (Slaughter y Leslie, 2001), ahora los académicos utilizan estas plataformas digitales definidas como «Facebooks académicos» bajo el lema establecido por Duffy y Pooley (2017) de «promocionarse o perecer».

\section{Referencias bibliográficas}

Academia.edu (2020). About Academia.edu. Recuperado de: https://www.academia. edu/about

Adell, J., Castañeda, L. \& Esteve, F. (2018). ¿Hacia la Ubersidad? Conflictos y contradicciones de la universidad digital. RIED. Revista Iberoamericana de Educación a Distancia, 21(2), 51-68.

Almetric (2020). What are altmetrics? Capturing the online attention surrounding scholarly content. Recuperado de: https://www.altmetric.com/about-altmetrics/ what-are-altmetrics/

Amigot, P. \& Martínez, L. (2013). Gubernamentalidad neoliberal, subjetividad y transformación de la universidad. La evaluación del profesorado como técnica de normalización. Athenea Digital, 13(1), 99-120.

Ball, S. J. (2012). Performativity, commodification and commitment: An I-spy guide to the neoliberal university. British Journal of Educational Studies 6o(1), 17-28.

Barnett, R. (1996). The evaluation of the higher education system in the United Kingdom. En R. Cowen (Ed.), The world yearbook of education: The evaluation of higher education systems: Londres: Kegan Paul.

Beer, D. (2015). Productive measures: Culture and measurement in the context of everyday neoliberalism. Big Data and Society, 2(1), 1-12.

Beer, D. (2016). Metric power. Londres: Palgrave Macmillan.

Brunner, J. J., Labraña, J., Ganga, F. \& Rodríguez-Ponce, E. (2019). Idea moderna de universidad. De la torre de marfil al capitalismo académico. Educación XXI, 22(2), 119-140. 
Burrows, R. (2012). Living with the h-index? Metric assemblages in the contemporary academy. The Sociological Review, 6o, 355-372.

Cannizzo, F. (2015). Academic subjectivities: Governmentality and self-development in higher education. Foucault Studies, 20, 199-217.

Castro-Gómez, S. \& Grosfoguel, R. (eds.). (2007). El giro decolonial: Reflexiones para una diversidad epistémica más allá del capitalismo global. Bogotá: Pontificia Universidad Javeriana/Siglo del Hombre Editores.

Cowen, R. (1996). Performativity, Post-modernity and the university. Comparative Education, 32(2),245-258.

De Angelis, M. \& Harvie, D. (2009). Cognitive capitalism and the rat-race: How capital measures immaterial labour in British universities. Historical Materialism, 17(3), 3-30.

Díez-Gutiérrez, E. J. (2018). Universidad e investigación para el bien común: la función social de la Universidad. Aula abierta, 47(4), 395-404.

Digital Science (2020). Our Portfolio. Recuperado de: https://www.digital-science.com/ products/

Duffy, B. E. \& Pooley, J. D. (2017). "Facebook for Academics": The convergence of selfbranding and social media logic on Academia.edu. Social Media+Society, 3(1), 1-11.

European Comission. (2020). The Digital Economy and Society Index (DESI). Recuperado de: https://ec.europa.eu/digital-single-market/en/desi

Fardella-Cisternas, C., Sisto-Campos, V., \& Jiménez-Vargas, F. (2017). La transformación de la universidad y los dispositivos de cuantificación. Estudios de Psicologia (Campinas), 34(3), 435-448.

Foucault, M. (1988). The history of sexuality. Vol. 3. The care of the self. New York: Vintage.

Foucault, M. (2007). Security, territory, population: Lectures at the Collège de France 1977-1978. Basingstoke: Palgrave Macmillan.

Foucault, M. (2008). The birth of biopolitics: Lectures at the Collège de France 19781979. Basingstoke: Palgrave Macmillan.

Fuchs, C. (2019). Karl Marx in the Age of Big Data Capitalism. En D. Chandler, D. \& C. Fuchs (eds.), Digital Objects, Digital Subjects: Interdisciplinary Perspectives on Capitalism, Labour and Politics in the Age of Big Data (pp. 53-71). London: University of Westminster Press.

Fuchs, C. \& Mosco, V. (eds.). (2015). Marx in the age of digital capitalism. Leiden: Brill.

Gómez, L., Bravo, M. J., \& Jódar, F. (2015). Gubernamentalidad neoliberal y producción de conocimiento en la universidad: genealogía de una configuración subjetiva. Universitas Psychologica, 14(5), 1735-1750. 
González-Calvo, G. \& Arias-Carballal, M. (2018). Effects from Audit Culture and Neoliberalism on University Teaching: An Autoethnographic Perspective. Ethnography and Education, 13(4), 413-427.

Grek, S. (2009). Governing by numbers: the PISA 'effect' in Europe, Journal of Education policy, 24(1), 23-37.

Hall, G. (2015, October 18). Does Academia.edu meanopen Access is becoming irrelevant? Media Gifts. Retirado de: http://www.garyhall.info/journal/2015/10/18/doesacademiaedu-mean-open-access-is-becoming-irrelevant.html

Hall, G. (2016). The uberfication of the university. Minneapolis, MI: University of Minnesota Press.

Harzing, A. W. (2010). The publish or perish book. Melbourne: Tarma Software Research.

Hirsch, J. E. (2005). An index to quantify an individual's scientific research output. Proceedings of the National Academy of Sciences, 102(46), 16569-16572.

Jessop, B. (2017). Varieties of Academic Capitalism and Entrepreneurial Universities: On Past Research and Three Thought Experiments. Higher Education 73(6), 853870 .

Johnstone, D., Arora, A. \& W. Experton (1998). The Financing and Management of Higher Education: A Status Report on Worldwide Reforms. Nueva York: World Bank.

Kitchin, R. (2014). The data revolution: Big data, open data, data infrastructures \& their consequences. London: Sage.

Lander, E. (comp.). (2000). La colonialidad del saber: eurocentrismo y ciencias sociales. Perspectivas latinoamericanas. Buenos Aires: CLACSO.

Leite, D. (2010) Brazilian higher education from a post $\square$ colonial perspective. Globalisation. Societies and Education, 8(2), 219-233.

Lynch, K. (2013). New managerialism, neoliberalism and ranking. Ethics in Science and Environmental Politics, 13, 141-153.

Lupton, D. (2015). Digital sociology. Routledge: New York and London.

Lupton, D. (2016). The quantified self: A sociology of self-tracking. Cambridge: Polity Press.

Lupton, D., Mewburn, I. \& Thomson, P.(2018). The digital academic: Criticalperspectives on digital technologies in higher education. Milton Keynes: Routledge.

Lyon, D. (2018). The Culture of Surveillance: Watching as a Way of Life. Cambridge, MA: Polity Press.

Lyotard, J-F. (1984). The Postmodern Condition: A Report on Knowledge. Minneapolis: University of Minnesota Press.

Manzano-Arrondo, V. (2015). Academia, evaluación y poder. Revista de Sociología de la Educación-RASE, 8, 197-222. 
Mayer-Schonberger, V. \& Cukier, K. (2013). Big Data: A Revolution that Will Change How We Live, Work and Think. London: John Murray.

Means, A. J. (2018). Learning to save the future: Rethinking education and work in an era of digital capitalism. New York: Routledge.

Mignolo, W. D. (2011). The Darker Side of Western Modernity: Global Futures, Decolonial Options. Durham, NC: Duke University Press.

Mirrlees, T., \& Alvi, S. (2019). EdTech Inc.: selling, automating and globalizing higher education in the digital age. Routledge: London and New York.

OECD (1996a). Employment and Growth in the Knowledge-based Economy. Paris: OCDE.

OECD (1996b). Measuring what people know: human capital accounting for the knowledge economy. Recuperado de https://www.oecd-ilibrary.org/docserver/9789264065482en. + pdf? expires $=1592760927 \&$ id $=$ id\&accname $=$ guest\&checksum $=E 4984 B A 867 \mathrm{C}$ $\mathrm{AC}_{5} \mathrm{~B} 7 \mathrm{CE} 2093 \mathrm{~B} 76 \mathrm{~F}_{3} \mathrm{~F}_{9458}$

OECD (1996c). The knowledge-based economy. Recuperado de: https://www. oecd.org/officiald ocuments/publicdisplaydocumentpdf/?cote=OCDE/ GD\%2896\%29102\&docLanguage=En

OECD. (2020). Digital Economy. Recuperado de: https://www.oecd.org/sti/ieconomy/

Olssen, M. \& Peters, M. A. (2005). Neoliberalism, higher education and the knowledge economy: from the free market to knowledge capitalism. Journal of Education Policy, 20(3), 313-345.

Orduña-Malea, E., Martín-Martín, A., Delgado-López-Cózar, E. (2016). ResearchGate como fuente de evaluación cien ca: desvelando sus aplicaciones bibliométricas. El profesional de la información, 25(2), 303-310.

Pedró, F., Subosa, M., Rivas, A., \& Valverde, P. (2019). Artificial intelligence in education: Challenges and opportunities for sustainable development. Paris: UNESCO.

Peters, M. A. (2020). Digital socialism or knowledge capitalism? Educational Philosophy and Theory, 52(1), 1-10.

Peters, M. A. \& Jandrić, P. (2018). The Digital University: A Dialogue and Manifesto. New York: Peter Lang.

Power, M. (2000). The Audit Society - Second Thoughts. International Journal of Auditing, 4, 111-119.

Plum Analytics (2020). Plum Analytics/About/Leadership. Recuperado de: https:// plumanalytics.com/about/leadership/

Pritchard, A. (1969). Statistical Bibliography or Bibliometrics. Journal of Documentation 25(4), 348-349.

ResearchGate (2020). About us. Recuperado de: https://www.researchgate.net/about 
Rose, N. (1991). Governing by numbers: Figuring out democracy. Accounting Organization and Society, 16(7), 673-692.

Rose, N. (1999). Governing the soul: The shaping of the private self (2 ed.). London: Free Association Books.

Santos, B. de S. (2010). Epistemologias del sur. Siglo XXI: México.

Saura, G. (2020). Mobilising the philanthropic neoliberalisation of Teach For All in Spain. En M. A.M. Thomas, E. Rauschenberger y K. Crawford-Garrett (eds.) Examining Teach For All. International Perspectives on a Growing Global Network (pp. 138156). London and New York: Routledge.

Saura, G. \& Bolívar, A. (2019). Sujeto Académico Neoliberal: Cuantificado, Digitalizado y Bibliometrificado. REICE. Revista Iberoamericana sobre Calidad, Eficacia y Cambio en Educación, 2019, 17(4), 9-26.

Schiller, D. (2000). Digital capitalism: Networking the global market system. Cambridge: MIT Press.

Sellar, S. (2015). A feel for numbers: Affect, data and education policy. Critical Studies in Education, 56(1), 131-146.

Selwyn, N. (2016). Is technology good for education? Cambridge: Polity Press.

Shahjahan, R. A., G. Blanco Ramirez, \& V. D. O. Andreotti. (2017). Attempting to Imagine the Unimaginable: A Decolonial Reading of Global University Rankings. Comparative Education Review 61(1), 51-73.

Sharpe, M. \& Turner, K. (2018). Bibliopolitics: The History of Notation and the Birth of the Citational Academic Subject. Foucault Studies, 25, 146-173.

Shore, C. \& Wright, S. (2015). Audit culture revisited. Rankings, rantings, and the reassembling of society. Current Anthropology, 56(3), 421-444.

Slaughter, S. \& Leslie, L. L. (2001). Expanding and elaborating the concept of academic capitalism. Organization, 8(2), 154-161.

Slaughter, S., \& Rhodes, G. (2004). Academic Capitalism and the New Economy: Markets, State, and Higher Education. Baltimore: Johns Hopkins University Press.

Srnicek, N. (2017). Platform Capitalism. Hoboken: Wiley.

Sundararajan, A. (2016). The sharing economy: The end of employment and the rise of crowd-based capitalism. Cambridge: Mit Press.

Tanczer, L. M., Deibert, R, J., Bigo, D., Franklin, M. I., Melgaço, L., Lyon, D. Becky Kazansky, B., \& Milan, S. (2020). Online Surveillance, Censorship, and Encryption in Academia. International Studies Perspectives, 21(1), 1-36.

Torres-Salinas, D., Cabezas, A. \& Jimenez, E. (2013). Altmetrics: nuevos indicadores para la comunicación científica en la Web 2.o. Comunicar, 41, 53-60. 
Vayreda, A.; Conesa, E.; Revelles-Benavente, B. \& González-Ramos, A. M. (2019). Subjectivation processes and gender in a neoliberal model of science in three Spanish research centres. Gender, Work \& Organization, 26, 430-447.

Williamson, B. (2017). Big data in education: the digital future of learning, policy and practice. London: Sage.

Williamson, B. (2019). Datafication of education: a critical approach to emerging analytics technologies and practices. En Rethinking Pedagogy for a Digital Age (H. Beetham y R. Sharpe, R., pp. 212-226). London: Routledge.

Williamson, B., Bayne, S. \& Shay, S. (2020) The datafication of teaching in Higher Education: critical issues and perspectives. Teaching in Higher Education, 25(4), 351-365.

Wyatt-Smith, C., Lingard, B., \& Heck, E. (2019). Digital learning assessments and big data: Implications for teacher professionalism. UNESCO Education Research and Foresight Working Papers, 1-23.

Zuboff, S. (2019). The Age of Surveillance Capitalism: The Fight for a Human Future at the New Frontier of Power. New York: Public Affairs. 